\title{
A Fast Response Performance Simulation Screening Tool in Support Of Early Stage Building Design
}

\author{
Marco Picco ${ }^{1}$, Marco Marengo ${ }^{1}$ \\ ${ }^{1}$ University of Brighton, Brighton, UK
}

\begin{abstract}
This paper aims to introduce the development of a simplified dynamic building energy simulation screening tool aimed to support early stage building design and feasibility studies while considering the lack of resources typical of those stages of the design process. The structure and main characteristics of the tool are discussed, providing insight on how they can benefit the integrated design process of more sustainable buildings. Results generated by the tool are shown to be comparable with the results of the simplified models, proving to be useful in the integration of energy aspects during the initial stages of building design.
\end{abstract}

\section{Introduction}

For a few years now, we've seen the constant raise in popularity for the concept of zero carbon buildings (ZCB) and its various interpretations as one of the main approaches in order to reduce $\mathrm{CO}_{2}$ emissions in the construction sector. Although the definition of ZCBs can take many different shapes, focusing on carbon emissions (ZCB) or energy consumption (ZEB); accounting for onsite or off-site energy generation (NZEB) or allowing a certain level of energy consumption (nZEB), the movement toward more sustainable buildings is now apparent.

Most countries already have national or international regulations in place, such as Europe with the EPBD (European Parliament, 2010) and its recent amendment (European Parliament, 2018), or Japan with targets set for 2020 and 2030 (METI, 2010). Other countries are developing such policies with short term targets, such as China (Zhang, 2018). Finally, in countries where there is no push from governments, a bottom up approach can be seen, with independent organizations and virtuous local authorities setting the path for the adoption of such standards.

The trend toward ZCB is inevitably leading to an increase in complexity in the design and operation of buildings, pushing their performance to the limit and integrating multiple systems and technology (Athienitis et al., 2010). This consequently leads to the need of performance optimization, something that can only be achieved by adapting the design process by applying a more integrated approach, through what is commonly defined IDP, integrated design process, and the use of advanced tool such as building performance simulation, particularly in the phases when they can achieve the best results, such as the first phases of the design process (Ferrero et al., 2015).

A multitude of simulation tools are currently available, providing the users with the possibility of preforming advanced simulation to support the design process of the building, to name a few, EnergyPlus, ESP-R, IES-Ve and various others offer robust and validated code with a range of interfaces and output complexity. Nonetheless, many of such simulation tools are not suitable for the diffused integration of building performance simulation within the design process, particularly when looking at early stage design.

Two major obstacles are seen that prevent the implementation of BPS in those stages, the lack of appropriate tools and the lack of resources (Attia et al., 2013; Ostergard et al., 2016).

Lack of tools is self-explanatory, as there is a clear perception from the end-users that none of the tools currently available are suitable for them, lack of resources can instead be traced back to the significant amount of time and information required to develop a building model, perform simulations and post process the output in order to obtain useful results, and this becomes more and more relevant when trying to apply such tools in the earliest stages of building design, when the process needs to be quick and iterative, time is scarce, and most of the information required for the use of complex tools has not been defined yet. This is not to the fault of the available tools, as most of them are not designed to be used during early stage design, when resources are very limited, and are better suited for the later stages of building design when BPS can validate the decisions made and help in optimizing the operations of the building.

Other projects have attempted to tackle this issue, with various degrees of success. Available details on existing tools have been carefully analysed by the authors, coming to the conclusion that none is currently available and easily accessible that can provide a general solution for the integration of BPS in early stage design, as they are either too complex, only tackle specific aspects of the problem or are only partially validated, particularly in relation to the use of simplified modelling to describe complex cases.

Table 1 below shows a non-exhaustive list of some of the most promising tools identified during the research and for each provide a short list of the reasons why they are 
still considered not suitable for the implementation during early stage building design.

Table 1: List of Similar simulation tools considered

\begin{tabular}{|c|c|}
\hline $\begin{array}{l}\text { MIT Design } \\
\text { Advisor }\end{array}$ & $\begin{array}{l}\text { - Focused on a specific aspect, } \\
\text { - Limited on inputs, } \\
\text { - Strong model hypotheses, } \\
\text { - Partially validated } \\
\end{array}$ \\
\hline ZEBO b1 & $\begin{array}{l}\text { - Focused on a specific type of building, } \\
\text { - Not web-based }\end{array}$ \\
\hline Opt-E Plus & $\begin{array}{l}\text { - Focused on optimization, } \\
\text { - Not available to public, } \\
\text { - High time requirements due to the } \\
\text { number of simulations, } \\
\text { - Not web-based }\end{array}$ \\
\hline H.E.N.K. & $\begin{array}{l}\text { - Limits in the simulation code, } \\
\text { - Lack of information, } \\
\text { - Not available to public, } \\
\text { - Not web-based }\end{array}$ \\
\hline
\end{tabular}

Based on this initial assessment, what is needed in order to facilitate the implementation of BPS in the early stages of building design is a tool that is: readily accessible, easy to understand and use, fast, flexible enough to describe a significant number of cases, but at the same time able to deliver results within an acceptable accuracy range. This brief assessment does not take into account more detailed tools, such as DesignBuilder, Openstudio, IES-Ve as they are defined in order to be used in later stages of the building design.

\section{The proposed solution}

The proposed screening tool solves the above-mentioned dilemma by providing a quick and easy way to perform simplified energy simulation to evaluate different design options, fast enough to be used in early stage design while still able to provide useful information to fuel the decision process. The tool has no intention to substitute more complex flexible solutions already available on the market, such as EnergyPlus or Ies-Ve, but rather complement them covering stages in which the use of those tools cannot be justified.

In order to create such a tool, some essential steps have been taken including: the selection of a suitable simulation code, the definition of a simplified building description model, the definition of all logics required to automatically generate the simplified model, and the ones required to postprocess the results into a usable form, and the definition of a series of databases containing the information required to run the simulation usually unavailable during early stage design. Lastly, all the required infrastructure and the various interfaces have been developed in order to provide the user with an easily accessible and user-friendly tool.

The first step of the project has been the selection of the most suitable simulation code to support the definition and testing of the building description model and finally to be integrated in the proposed platform. Various codes have been initially considered and used to test the developed models, but finally the authors selected the well-known and renowned EnergyPlus as the underlying simulation engine (Crawley et al., 2004; Henninger et al., 2010).

A conceptualized scheme of the workflow and structure used for the development of the platform can be seen in Figure 1, highlighting an additional novelty aspect of the proposed tool, its nature as a web platform, as the only the input and results interfaces, identified as front end, are processed by the user device, while all backend processes, such as model generator, simulation, and postprocessing, are taken care of by the server.

The definition of the building description model has been discussed in detail in previous publication by the authors

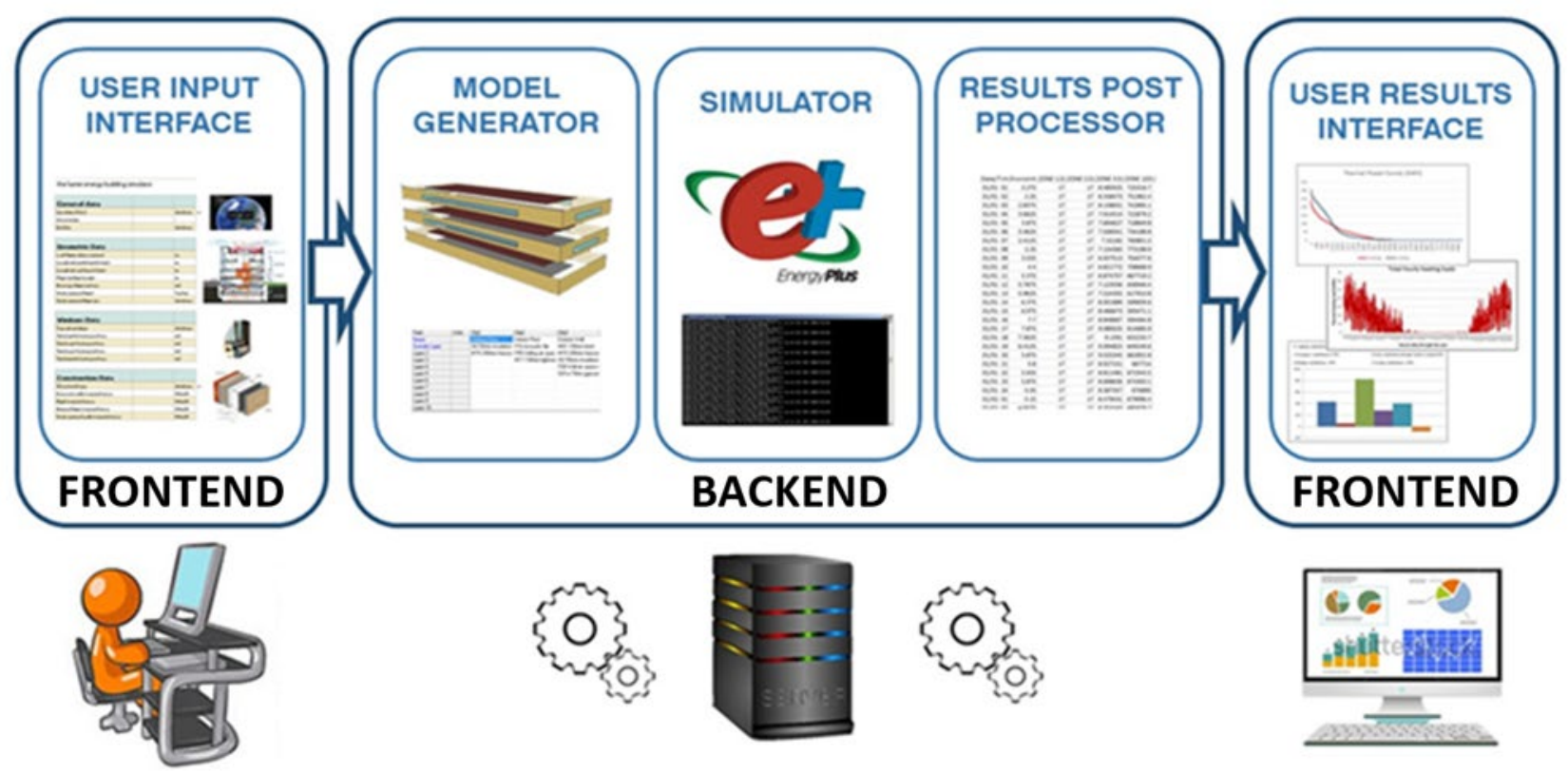

Figure 1: Workflow of the tool and its subsystems 
(Picco et al., 2015). The simplified model has been obtained by applying a series of well-defined simplifications steps to a series of detailed models in order to identify the main sources of deviation on the selected outputs. The result of this process is a simplified description model that was subsequently implemented in the proposed tool. Interesting to be noted, between the eight defined simplification steps, two simplifications generating the most significant deviations are the ones related to the thermal zoning of the buildings and to the operational parameters of the various floors such as internal loads and scheduling. Arguably, those two aspects are the ones that prove to be most difficult to correctly take into account in any simulation, and even more in the early stages of the design of the building, when it is expected available details and resources would be insufficient in order to correctly model them at an appropriate level of detail, often resulting in simplified zoning of the building and the need to make strong assumptions on the operations of the building. Conversely, all steps related to the geometric definition of the model proved to be less significant in term of resulting deviation in the results.

Despite the numerous simplifications applied, all case studies analysed during the research show total deviations equal or below $16.2 \%$, (Picco, 2014) well below what was defined as an acceptable margin of accuracy of $20 \%$ at the beginning of the project. This threshold has been defined by the authors and, although not directly related, can be compared to the recommended acceptable value of mean bias error for detailed and calibrated simulation models as presented in the Measurement and Verification guidelines from DOE (DOE, 2008) and ASHRAE guidelines (2014) equal to $10 \%$.

Despite the simplicity and rapidity, results are given with an acceptable margin of accuracy compared to detailed simulations. The proposed tool will help the design process evolve toward an integrated approach and adapt to the foreseeable changes in regulations and market demand of low- and zero-carbon buildings.

The structure of the tool, the interfaces and the databases are further discussed in the following chapters.

\section{The Tool}

The proposed tool is a fully web-based platform, currently available in closed alpha stage to selected users and accessible through any of the major web browsers. As only the interfaces need to be processed by the user device, it is envisioned the platform will be accessible from any device with only minor adaptations, including tablets, smartphones and other operative systems, for which none of the simulation codes may even be available.

Once the user inputs all the required information in the input interface, the inputs are packaged and sent to the server, the model generator proceeds to read them, collect the additional required details and creates the simulation model, EnergyPlus is then used to run the simulation. Output from the simulation code is postprocessed and the obtained results are sent back to the user interface for display in automatically generated graphs and charts ready to be used to inform the design process.

The previously defined description model has been fully implemented in the discussed screening tool without any significant alteration aside of the minor required adaptation in order to allow a more streamlined automatic generation of the model, as discussed below while analysing the required computational logics. As shown in the results section, those minor changes do not have any significant impact in the generated results. A detailed list of all inputs required for the simplified model has been previously published in a separate paper by the author (Picco et al., 2016). The next four sections will analyse each one of the four crucial components that allow the tool to operate and their impact in the facilitation of the use of building performance simulation during early stage design.

\section{The Input Interface}

The first element the user will come into contact with is the input interface. It is essential for the interface to be as user-friendly as possible, easy both to understand and use, in order to allow the average user to access the potential benefits that building performance simulation might bring.

It is expected for the average user to have a general understanding of the thermal behaviour of buildings and the underlying physics and terminology, while not necessarily being comfortable in using complex simulation tools. Required information needs to be clearly displayed and explained to the user, for this reason, the 25 entries required in order to perform the simulation have been divided in five categories: General, Geometry, Windows, Construction and HVAC. Figure 2 below provides an example of the displayed interface in its current form, showing the Geometry entry section.

The input process has also been streamlined, to guide the user through the input phase. Each entry field is characterized by a clear name and associated help function. Numeric fields also display unit, minimum and maximum values, default values already placed in the required fields and buttons to increase and decrease the value. Database selection fields instead allow the user to access dropdown menus to select the correct entry.

The location database is an exception as, to streamline the process and help the user in selecting the most appropriate weather file, the selection can be made with the support of a map displaying all available weather data with its relative positioning on the map.

Default values, where appropriate, can immediately help the user gaining a better understanding of the required input and provide a useful resource when no information is available for the current project, although all data entries have been defined in order to make this eventuality as rare as possible, as the level of detail required for the screening tool should always be available at any stage of the design process. 


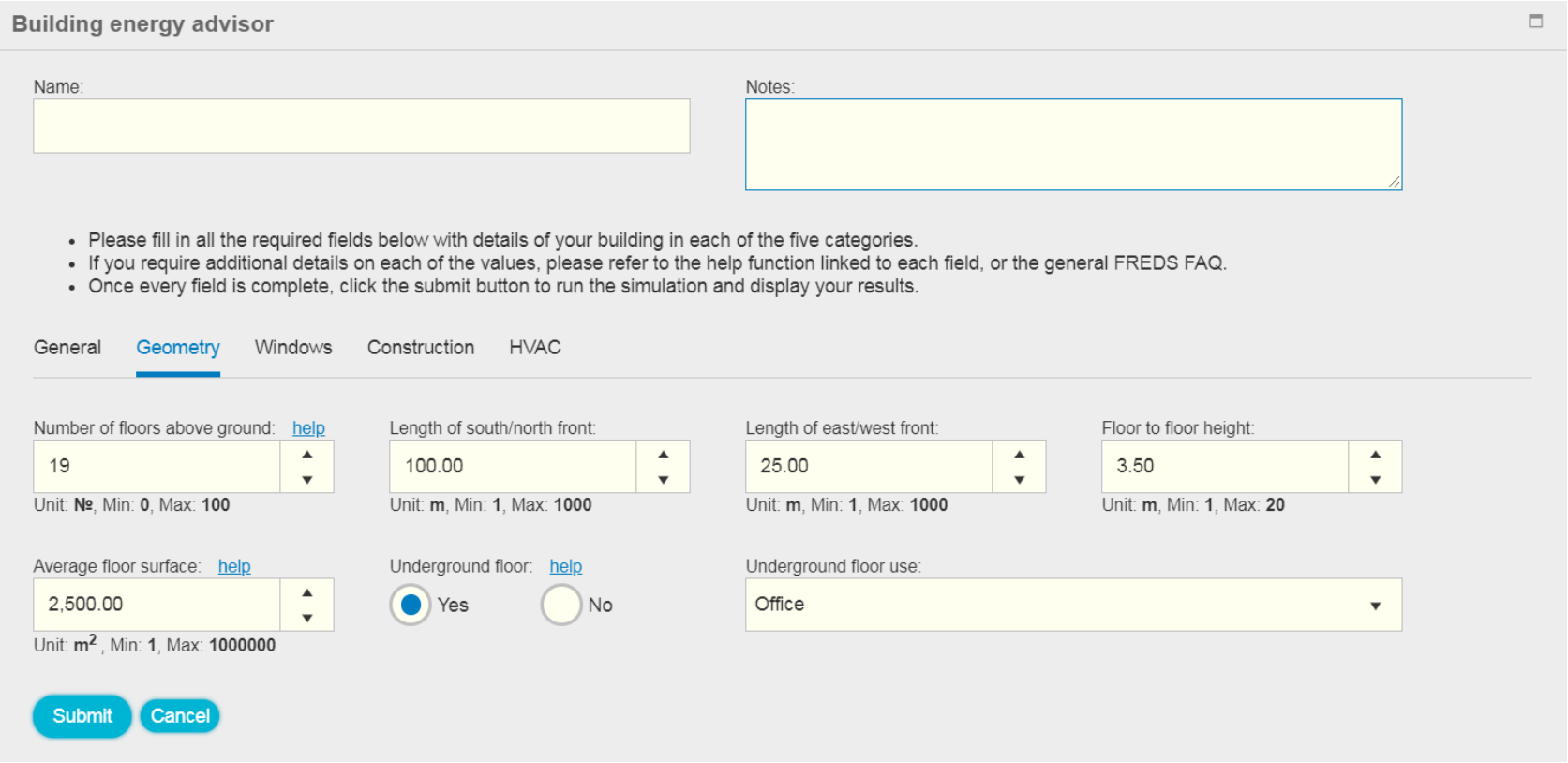

Figure 2: Input Interface Example

The help function is essential to provide the user with a clear definition of the required data and how to retrieve it if needed, additionally a list of typical values can be provided to further help in case the information is not available or not yet defined, providing a useful device to test the impact of variations in such values following available guidelines.

Displaying maximum and minimum values is also useful in order to avoid or limit gross errors and to perform an initial automatic check while the model is being generated.

\section{The Automated model generation}

Possibly the most complex part of the development of the platform has been the development of all the required code to ensure the collected data coming from the interface is adequately processed and used to generate a suitable building description model to be simulated through EnergyPlus. This does not include all code that necessarily needs to be developed for the functioning of the web-platform itself, which is in its own way an extensive and complex piece of work, although more akin to the field of computer science, but only the rules and algorithms that have been defined and implemented in order to be able to automatically run a simulation starting only from the entries required in the input interface.

A series of template files have been defined, containing all the static information that needs to be included in an EnergyPlus input data file (IDF), with one being the main starting template and the others being combined in order to generate all possible models that are currently supported by the tool. Those templates also contain all the required connections to the rules and algorithms developed to allow for dynamic information coming from the interface to be included in the model, with over 100 connection points between the different templates and the main template file being almost 1000 lines long.
The entirety of the code developed in order to translate all the information provided by the input entries to the dynamic fields included in the templates amount to a total of 1241 lines, defined following principles of robustness, understandability, reliability and maintainability and carefully tested in order to avoid any foreseeable errors that would result in an unusable IDF model for EnergyPlus, or even worse, an incorrect one, leading to unreliable results.

The majority of such rules relate to the translation of numerical values entered by the user to the connected numerical values that need to be included in the input data file, ranging from surface coordinates, to material properties, to schedule values.

In addition to that, a portion of such rules relates to the loading of additional information from one of the five defined databases, themselves containing additional rules and connections for dynamic fields where necessary. The five databases are further detailed in the following subchapter.

Finally, additional functionalities have been included in the platform in the form of a backend interface allowing easy maintenance of the code and database, including its continuous development and expansion and pre-emptive testing of any new entry from the development team.

\section{The Databases}

Databases are one of the essential components of the tool, as they contain all the information that is usually not available during early stage design at the level of detail require to perform dynamic simulations, or would otherwise require too much time to input in the simulation model, making the results useless in the context of integrated design.

Although the detailed content of the databases is not discussed in this paper, as it will be object of separate publications, a brief overview of the various databases is provided below. 
In its current form, five databases have been implemented in the platform: Location, End Use, Underground End Use, Structural type and Type of Windows. Each database has been implemented following the concepts of robustness, modularity and flexibility. New entries can be easily added to each database and/or modified as required through the previously mentioned backend interface. Before becoming available to the end user, each is automatically tested to ensure no errors are generated.

\section{Results Interface}

Final step of the proposed workflow is the display of readily useable results to the user. This is delivered by the combination of the results postprocessor, managed in the backend of the platform by the server, and the results display interface, located on the user device.

Results coming from the simulation engine, are usually not readily usable in the design process, and they need to be processed by a building performance simulation expert in order to be a useful input to the design process, this poses an obstacle to the proposed approach and therefore a post processing phase has been defined.

This is even more relevant as the use of a simplified model poses the need to interpret the results based on the assumptions used in the definition of the simplified model itself, and therefore an unexperienced user might find additional obstacles in gaining useful insight from interpreting the direct output from the simulation code, all assumptions are instead taken into account by the result post-processor, delivering readily usable information.

The result post-processor takes the csv file generated by EnergyPlus as an input, generates all the values required to populate the predisposed result charts and send them to the user device to use in the results display interface.
In its current form, the platform displays results in four separate charts directly within the web interface, additionally each chart can be downloaded in various formats. Each chart is not only displayed as a picture, but is instead a fully interactive chart, users can highlight the preferred results, interrogate the chart to immediately obtain hourly or daily values and change the displayed timeframe of yearly chart to increase readability. Report Timestep of results in the displayed charts is automatically adapted to the requested timeframe, ranging from hourly for a day timeframe to daily values for yearly view.

Figure 3 below shows the first of those charts, representing the thermal energy needs of the buildings throughout the year, both in term of heating and cooling, useful to understand the behaviour of the building throughout the year.

Other displayed results include the thermal power curves of the building, to identify the energy loads on the required plant, the maximum required capacity expected and the number of hours a certain capacity load is exceeded throughout the year, useful to pre-size the thermal plants in the building and understand the impact of different technologies and sizing options. Temperature distribution throughout the year for an average room within the building is also displayed in a separate chart at hourly level to allow user to gain insight on the comfort conditions within the building and how it behaves throughout the year when compared to the outdoor temperature considered for the simulation.

Lastly, a summary of the energy needs of the building, on monthly basis is displayed in a chart and associated table to allow immediate understanding of the energy needs for heating and cooling throughout the year and allow easy

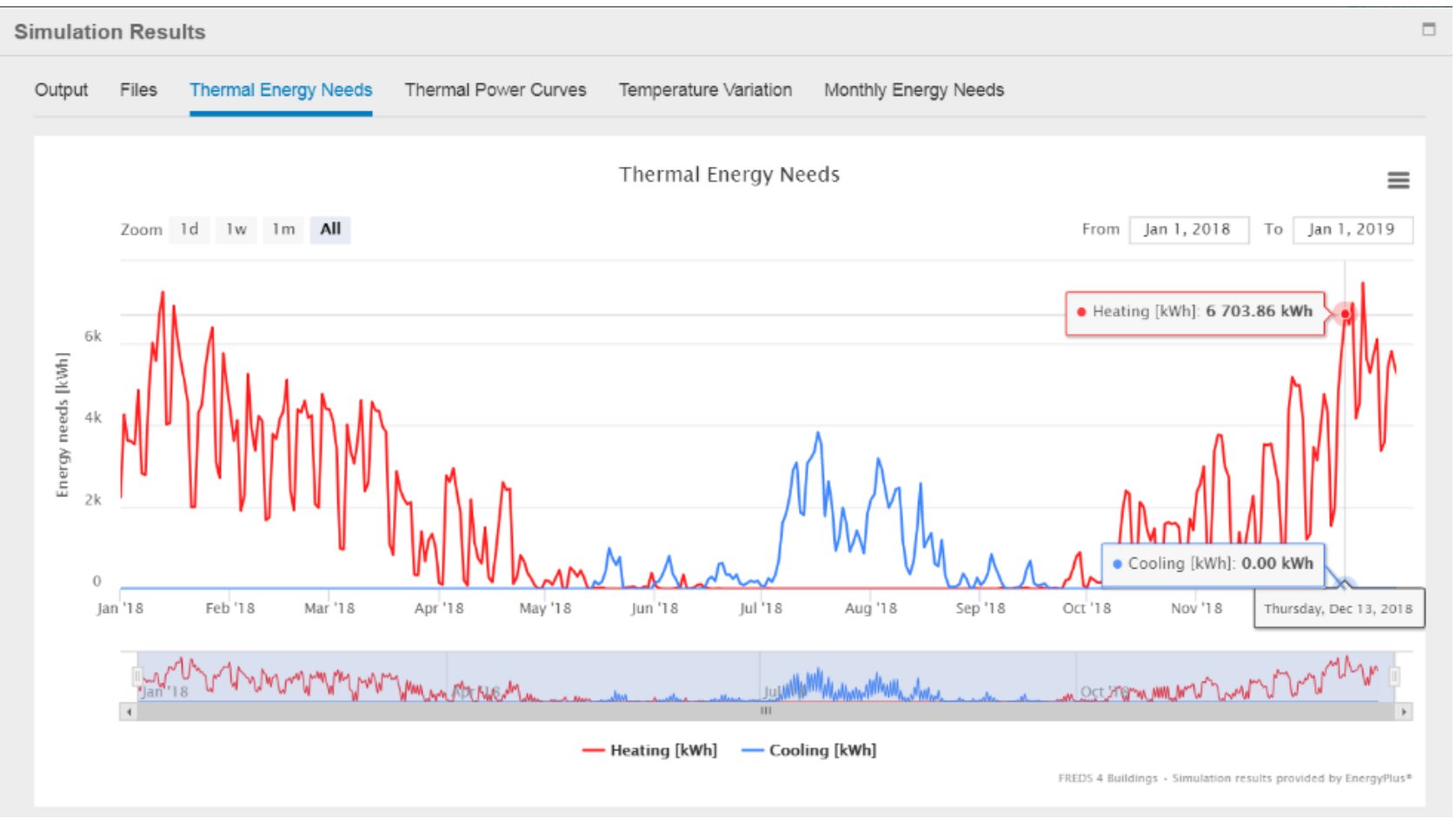

Figure 3: Example of Results on daily timestep 
and quick comparisons between different design options, as shown in Figure 4.

Additional options to allow the user to directly download the output files generated as an output by EnergyPlus, a summary of the entries received by the model generator, and the output of the result post-processor have been included in the platform, although access to such files to all users is currently being evaluated as, due to the previously mentioned underlying assumptions required to interpret the results, this might generate unneeded confusion and reduce the usability of the platform.

\section{Overview of Results}

As an example of application of the tool, one of the previously developed case studies, used for the validation of the simplified model descriptor, has been simulated through the final version of the proposed web platform, some deviation in results is expected compared to the simplified model simulations, previously run manually in EnergyPlus, due to the adaptations to the description model required to streamline the automatic generation of the model, and the generalization of the database entries to be used in the web platform such as the building end use and structural type.

Table 2 below shows the input entries used to perform the simulation through the developed interfaces, the building is a medium size private clinic located in Bergamo, Italy, with an approximate total floor area of $5000 \mathrm{~m}^{2}$ distributed on 5 floors, of which one below ground, below average thermal characteristics of the envelope and complex floor shape. Due to the nature of the model, all required information was available and therefore none of the entries was kept to its default values.

For the purpose of this paper, total heating and cooling energy needs for the building are taken into consideration as the parameters to compare between the different models. Absolute values for those parameters can be seen in Table 3 below.

Table 2: Input data for example of application

\begin{tabular}{|l|c|l|}
\hline \multicolumn{1}{|c|}{ Entry id } & Value & \multicolumn{1}{|c|}{ Unit } \\
\hline location_city_id_input & Bergamo [IT] & - \\
\hline orientation & -70.8 & $\circ$ \\
\hline end_use & Hospital & - \\
\hline$n$ floors_above_ground & 4 & - \\
\hline length_east_west_front & 53.4 & $m$ \\
\hline length_south_north_front & 83.5 & $m$ \\
\hline floor_to_floor_height & 3.5 & $m$ \\
\hline average_floor_surface & 1124 & $\mathrm{~m}^{2}$ \\
\hline undergroundfloor & Yes & - \\
\hline underground_floor_use & Hospital & - \\
\hline type_of_windows & Double Clear & - \\
\hline total_east_facing_surface & 59.1 & $\mathrm{~m}^{2}$ \\
\hline total_south_facing_surface & 230.4 & $\mathrm{~m}^{2}$ \\
\hline total_north_facing_surface & 130.3 & $\mathrm{~m}^{2}$ \\
\hline total_west_facing_surface & 125.0 & $\mathrm{~m}^{2}$ \\
\hline structural_type & Masonry & - \\
\hline roof_transmit & 1.729 & $\mathrm{~W} / \mathrm{m}^{2} \mathrm{~K}$ \\
\hline external_walls_transmit & 1.570 & $\mathrm{~W} / \mathrm{m}^{2} \mathrm{~K}$ \\
\hline ground_floor_transmit & 1.348 & $\mathrm{~W} / \mathrm{m}^{2} \mathrm{~K}$ \\
\hline underground_walls_transmit & 1.570 & $\mathrm{~W} / \mathrm{m}^{2} \mathrm{~K}$ \\
\hline$t$ heating_setpoint & 22.5 & ${ }^{\circ} \mathrm{C}$ \\
\hline$t$ cooling_setpoint & 28.0 & ${ }^{\circ} \mathrm{C}$ \\
\hline$t$ heating_setback & 18.0 & ${ }^{\circ} \mathrm{C}$ \\
\hline$t$ cooling_setback & 28.0 & ${ }^{\circ} \mathrm{C}$ \\
\hline heatrecovery_efficiency & 0.0 & $\%$ \\
\hline The & & \\
\hline
\end{tabular}

The detailed model is defined as a highly detailed model developed for the case study, including highly detailed geometry, zoning, material properties, and internal loads distribution, calibrated based on monitored energy consumption form the building. The simplified model is defined as the end result of the simplification process, as

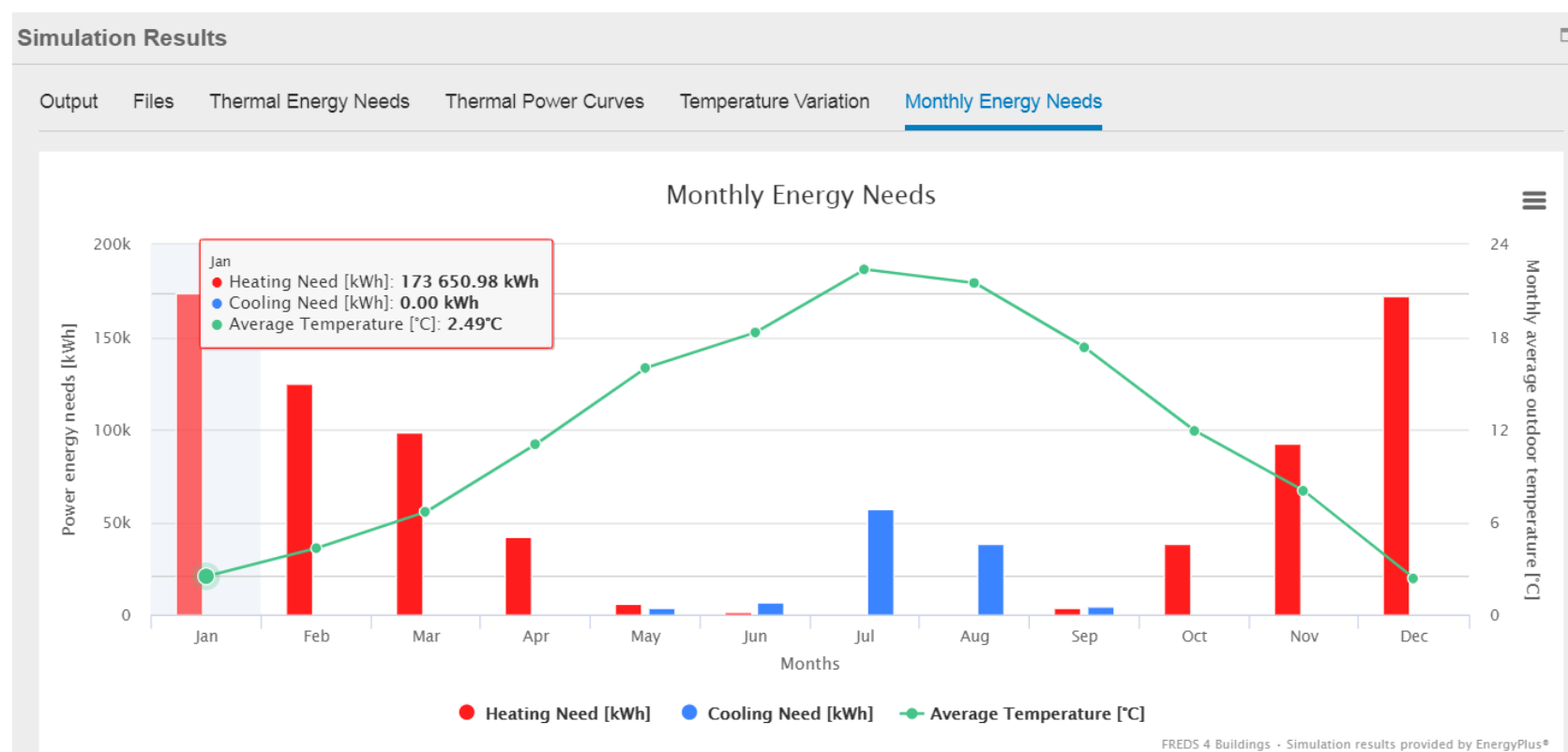

Figure 4: Example of Summary Results 
defined in previous papers by he author, defining the simplified model descriptor. Finally, the "Final Tool model" is the model automatically generated by the proposed tool, in all aspects similar to the Simplified model with the exception of few minor changes to streamline the model generation process.

Table 3: Summary of model outputs

\begin{tabular}{|l|c|c|}
\hline \multicolumn{1}{|c|}{ Model } & $\begin{array}{c}\text { Heating } \\
{[\mathbf{k W h}]}\end{array}$ & $\begin{array}{c}\text { Cooling } \\
{[\mathbf{k W h}]}\end{array}$ \\
\hline Detailed Model & 791,075 & 128,102 \\
\hline Simplified Model & 769,473 & 110,219 \\
\hline Final Tool Model & 753,134 & 110,076 \\
\hline
\end{tabular}

Results obtained by the final tool are comparable to the ones obtained by the Simplified model, settling a total deviation compared to the detailed model equal to $4.8 \%$ for the Heating loads and $14.0 \%$ for the cooling loads, differences between simplified model, manually modelled in EnergyPlus, and final tool model, automatically generated by the platform, are in the range of $2 \%$ in the in the worst case, and related to the definition of geometric elements to facilitate the automatic generation of the model, most relevant of which being the definition of the dimensions of roof and ground floor. Both results are considered acceptable as within the previously established accuracy margin of $20 \%$.

\section{Conclusion}

Building performance simulation is a fundamental instrument to support the design of new buildings thanks to its ability to allow a better understanding of the expected performances of different design options. The constant increase in standards and necessity of designing increasingly efficient buildings is making it a necessity to integrate such analyses in the design process of buildings, with a particular focus at the initial stages of building design, when the most impact can be achieved. This approach is still not widespread in the industry, due to the lack of resources and suitable tools.

The proposed platform aims to tackle this issue by providing a way to integrate building performance simulation in the design process, particularly during early design, thanks to its easiness of use, limited time requirements and ability to perform the analyses with a limited number of inputs, compatible to what might be available at that stage of the design.

Thanks to the use of a validated simulation code, coupled with the developed and tested simplified description model, the final tool is capable of delivering results with limited deviation compared to a detailed model of the building, below what has been defined as an acceptable accuracy margin of $20 \%$, as shown in this paper and previously published case studies.

Based on the first observed results and collected feedback from industry, the authors believe that the proposed solution will have a significant impact in facilitating the integration of building performance simulation in the design process of building, with a particular focus on early stage design and feasibility studies, providing an instrument to obtain useful information to fuel the design process and by consequence allowing the industry to move toward the constant improvement in building performances.

Additionally, the platform has the great potential to be used as an educational tool, allowing both students and the general public to better understand the thermal behaviour of buildings and the impact of different design choices while requiring a limited initial level of knowledge, providing an approachable entry point to building performance simulation concepts.

\section{Acknowledgement}

The authors want to thank and acknowledge all the partners that contributed to the development of the tool in its current state, including but not limited to Mr. Gaetano Fabio Graziano and Mr. Giorgio Ghisalberti.

This research was partially supported by the Proof of Concept Innovation fund from the department of "Research, Enterprise and Social Partnerships" of the University of Brighton.

\section{Nomenclature}

$Z C B$, Zero Carbon Buildings;

$Z E B, \quad$ Zero Energy Buildings;

$N Z E B$, Net Zero Energy Buildings;

$n Z E B$, Nearly Zero Energy Buildings;

$E P B D$, European Performance of Buildings Directive;

$I D P, \quad$ Integrated Design Process;

$B P S$, Building Performance Simulation;

$I D F, \quad$ Input Data File;

CSV, Comma Separated Value;

\section{References}

ASHRAE (2014). ASHRAE Guideline 14-2014: Measurement of Energy Demand, and Water Savings. 2014.

Athienitis, A., Attia, S. (2010). Strategic design, optimisation, and modelling issues of net-zero energy solar buildings. Proceeding of EuroSun 2010, Graz, Austria

Attia, S., Gratia, E., De Herde, A., Hensen, J. (2012). Simulation-based decision support tool for early stages of zero-energy building design. Energy and Buildings, volume 49, 5-12.

Crawley, D.B., Lawrie, L.K., Pedersen, C.O., Winkelmann, F.C., Witte, M.J., Strand, R.K., Liesen, R.J., Buhl, W.F., Huang, Y.J., Henninger, R.H., Glazer, J., Fisher, D.E., Shirey, D.B., Griffith, B.T., Ellis, P.G., Gu, L. (2004). EnergyPlus: An Update, SimBuild 2004, Building Sustainability and Performance Through Simulation, Boulder, CO, USA, August 4-6, 2004.

DOE U S. M\&V Guidelines: Measurement and Verification for Federal Energy Project, Version 3.0, 2008. 
European Parliament (2010). Directive 2010/31/EU of the European parliament and of the council, of 19 May 2010 on the energy performance of buildings (recast).

European Parliament (2018). Directive (EU) 2018/844 of the European Parliament and of the Council of 30 May 2018 amending Directive 2010/31/EU on the energy performance of buildings and Directive 2012/27/EU on energy efficiency.

Ferrero, A., Lenta, E., Monetti, V., Fabrizio, E., Filippi, M. (2015). How to apply building energy performance simulation at the various design stages: a recipes approach. 14th IBPSA conference, Hyderabad, India, Dec. 7-9, 2015

Henninger R.H., M.J. Witte (2010). EnergyPlus Testing with Building Thermal Envelope and Fabric Load Tests from ANSI/ASHRAE Standard 140-2007. U.S.D.o. Energy, Editor, Washington DC.

Hensen, J. (2004). Towards more effective use of building performance simulation in design. 7th International Conference on Design \& Decision Support Systems in Architecture and Urban Planning, Eindhoven, July 25,2004

Østergård, T., Jensen, R.L., Maagaard, S.E. (2016). Building simulations supporting decision making in early design - A review. Renewable and Sustainable Energy Reviews, Volume 61, Pages 187-201.

Picco, M., (2014). Dynamic energy simulation toward integrated design of non-residential buildings. Model description simplifications and their impact on simulation results. Università degli studi di Bergamo, Bergamo, (Italy).

Picco, M., Marengo, M. (2015). On the impact of simplifications on building energy simulation for early stage building design. Journal of Engineering and Architecture, Volume 3 Issue 1

Picco, M., Marengo, M. (2016). Fast Response Building Energy Simulation in support of Low Carbon Design and Retrofit. Zero Carbon Buildings Today and in the Future 2016, September 2016, Birmingham, UK.

Bambardekar, S., Poerschke, U. (2009). The architect as performer of energy simulation in the early design stage. 11th International IBPSA Conference Building Simulation 2009, Glasgow, United Kingdom, 27 July

METI, Ministry of Economy, Trade and Industry (2010). Energy saving technology strategy 2011. Promotion of Zero Energy Building (ZEB) and Zero Energy Houses (ZEH).

Negendahl, K. (2015). Building performance simulation in the early design stage: An introduction to integrated dynamic models. Automation in Construction, Volume 54, Pages 39-53

Zhang, Y., Kang, J., Jin, H. (2018). A Review of Green Building Development in China from the Perspective of Energy Saving. Energies 11(2):334. 